Lingua Litteria Journal

\title{
L'Acte de Parole Directif dans la Bande-Dessinée (BD) Astérix Chez les Helvètes par René Goscinny et Albert Uderzo
}

\section{Mela Rahmayanti ${ }^{\bowtie}$ Isfajar Ardinugroho}

Département de la Langue et la Littérature Étrangère, Faculté des Langues et des Arts, Universitas Negeri Semarang, Indonesia

\begin{tabular}{l}
\hline Info d'article \\
\hline Histoire de l'Atrticle: \\
Reçu Janvier 2020 \\
Accepté Mars 2020 \\
Publié Mei 2020 \\
\hline Keywords: \\
L'acte de parole, les types \\
l'acte de parole, les formes \\
l'acte de parole
\end{tabular}

\begin{abstract}
\section{Extrait}

Les actes pris par les humains quand se divise prononcent ce discours est appelé des actes de parole. L'actes de parole a divisé en trois types, ces sont l'acte de parole locutoire, l'acte de parole illocutoire, et l'acte de parole perlocutoire. L'actes de parole illocutoire a divisé en cinq types, ces sont l'acte de parole assertif / représentatif, directif, permissif, expressif et déclaratif. Cette recherche a pour objectif de décrire 1) les types l'acte de parole directif dans la bande dessinée Astérix Chez Les Helvètes, 2) les formes l'acte de parole directif dans la bande dessinée Astérix Chez Les Helvètes. Le sujet de cette recherche est toutes les paroles dans la bande dessinée Astérix Chez Les Helvètes. L'objet de cette recherche est toutes les paroles qui sont des actes de paroles directifs dans la bande dessinée Astérix Chez Les Helvètes. La méthode utilisée à collecter les données est la méthode lire attentivement (simak). Cette méthode est faite en utilisant la technique de taraudage de langue (sadap), ensuite utilise la technique non participé à la conversation réelle (SBLC) et la technique catat (le chercheur a obtenu les données de prise de notes dans la carte de données). Dans cette recherche, la chercheuse utilise la méthode d'appariement pragmatique (metode padan pragmatis) pour analyser les données. Dans ce cas la chercheuse utilise la technique de base la technique divisé des éléments différencier (PUP). Ensuite la chercheuse se sert de la technique avancée de la technique de relier-comparer-égaliser (Hubung Banding Menyamakan). Sur la base des résultats de l'analyse, les types d'acte de parole directif peuvent être classés en 1) demandé (5données), 2) questionné (14 données), 3) exigé (25 données), 4) interdiction (2 données), 5) permissif (1 donné), 6) conseil (4 données). À partir des résultats de l'analyse, les forme des actes de parole peut être classée en: 1) l'actes de parole directif direct littéral (44 données), 2) l'actes de parole directif indirect littéral (3 données), 3) l'actes de parole directif direct non-littéral (1 donnée), 4) l'actes de parole directif indirect non-littéral (3 données).
\end{abstract}

(C) 2020 Universitas Negeri Semarang

${ }^{\boxplus}$ Addresse:
Gedung B4 FBS Universitas Negeri Semarang
Kampus Sekaran, Gunung pati, Semarang, 5022

ISSN 2252-6730

Kampus Sekaran, Gunung pati, Semarang, 50229 


\section{INTRODUCTION}

La langue comme l'outil de la communication est important pour exprimer les messages, les intentions ou les idées, parmi les gens. La manière de transférer les messages ou les intentions peut être exprimée directement ou indirectement. Ces manières existe ont pour but d'aider les gens à bien comprendre les intentions et leur contextes. Le contexte est l'un des facteurs de la communication. Tout les types communication se dépendent des contextes. La communication entre les personnes est non seulement faite à travers des mots, mais aussi on utilise le geste par des actes et des comportements. Les actes pris par les humains quand ils s'énoncent ce discours est appelé des actes de parole. Searle (cité par Wijana, 1996: 17 -22) a classifié un énoncé en trois actes, ces sont les locutoire, les illocutoire, et les perlocutoire. Wijana (1996: 18) les énoncés sont aussi avoir le but d'être utilisé pour faire ce qu'on appelle des actes de parole illocutoire. L'actes de parole illocutoire à diviser en cinq types, ces sont l'acte de parole assertif / représentatif, directif, permissif, expressif et déclaratif.

À partir de cette problématique la chercheuse veut analyser l'acte directif dans la bandedessinée (BD) Astérix Chez Helvètes. La chercheuse choisi ce BD comme l'objet de la recherche car ce BD est unique et faire la chercheuse être curieuse à le lire. Dans ce BD il y a beaucoup d'actes directifs, et ce BD utilise aussi la langue simple pour être facile à comprendre.

\section{MÉTHODE DE RECHERCHE}

Cette recherche utilise deux approches, ce sont l'approche théorique et l'approche méthodologique. L'approche théorique utilisée est la pragmatique. C'est-à-dire que la chercheuse comme l'analyseur considère les paroles dans le BD est l'acte de parole directif. L'approche méthodologique utilisé dans cette recherche est descriptive qualitative.

La méthode utilisée à collecter des données est la méthode lire attentivement (simak). Sudaryanto (2015:133) a dit que la méthode lire attentivement (simak) est la méthode fait en observant l'utilisation de la langue. Cette méthode est faite en utilisant la technique de taraudage de langue (sadap), ensuite utilise la technique non participé à la conversation réelle (SBLC). C'est la technique qui est fait en observant l'utilisation des langues sans la participation de chercheur dans la formation des données. En plus, cette recherche utilise aussi la technique avancée "noter " (catat) à l'ordinateur en classifiant par leurs contextes, le type d'acte de parole directif, la forme d'acte de parole et leur signification.

Dans cette recherche, la chercheuse utilise la méthode d'appariement pragmatique (metode padan pragmatis) pour analyser les données. Dans ce cas la chercheuse utilise la technique de base la technique divisé des éléments différencier (Pilah Unsur Penentu). Ensuite la chercheuse se sert de la technique avancée de la technique de relier-comparer-égaliser (Teknik Hubung Banding Menyamakan). C'est usé pour chercher la similarité des lexiques signifiés ayant de leurs fonctions respectives.

\section{RÉSULTAT ET DISCUSSION}

\section{Le type d'acte de parole directif}

Le type d'acte de parole directif dans cette recherche a divisé dans six types, ce sont demandé, questionné, exigé, interdiction, permissif, et conseil.

\section{Demandé}

Demandé s'exprime le désir du locuteur afin d'interlocuteur s'agit quelque chose. Voici l'explication :

1) CONTEXTE : LE SOIR AU PALAIS, LE GOUVERNEUR DE CONDATE FAISAIT LA FETE AVEC SES SOLDATS. ENSUITE, L'AGENT FINANCIER, CAIUS, EST 
VENU A VERIFIER LA COMPTABILITE EN PROVINCE. PUIS GAROVIRUS, LE GOUVERNEUR DE CONDATE A INVITE CAIUS A LE REJOINDRE POUR PROFITER DE LA FETE. MAIS, CAIUS A REFUSE CAR IL AVAIT ETE ENVOYE A CONDATE POUR S'EFFECTUER A SA TACHE, PAS POUR LE PLAISIR.

Garovirus: Avé, avé, Questeur! Tu nous surprends en pleine réunion familiale. Une petite fête entre intimes... Viens te joindre à nous. (ACLH: 5)

La parole (1) s'entre au type d'acte de parole directive avec l'intention d'inviter. La phrase "Viens te joindre à nous." Indique que cette parole directive est entrée à ce type (demandé) car Garovirus a directement invité Caius à rejoindre le parti.

\section{Questionné}

Questionné est une demande, dans ce type le locuteur demande des informations à son interlocuteur par les questions afin de donner certaines informations. Voici l'explication :

2) CONTEXTE: LE SOIR AU PALAIS DES CONDATE, CAIUS ETAIT MALADE D'UNE INTOXICATION ALIMENTAIRE. PUIS GAROVIRUS A APPELE LE MEDECIN POUR LE TRAITER. PUIS LE MEDECIN EST VENU EN DEMANDANT "OU SONT LES MALADES?" PUIS GAROVIRUS A AMENE LE MEDECIN A LA CHAMBRE.

Où est le malade? (ACLH: 9)

La parole (2) est un type d'acte de parole directive "questionné » ayant pour but de demander. La phrase indique que cette parole s'entre à ce type car le médecin demande directement aux malarias où le malade est Caius. La phrase utilise des phrases interrogatives.

Exigé

Dans l'acte de parole directif Exigé le locuteur veut que l'interlocuteur fasse quelque chose commandé. Dans ce cas le locuteur a un niveau d'autorité plus supérieur qu'interlocuteur. Mais, il est possible que l'énoncé de locuteurs ayant le même niveau d'autorité que l'interlocuteur soit un commande si les énoncés est prononcé avec un ton très aigu et à haute voix. On expliquera cidessous :

3) CONTEXTE : AU SOIR, LORSQUE GAROVIRUS PARTICIPE A UNE FETE AVEC SES SOLDATS. UN SOLDAT DEMANDE A GAROVIRUS DE RENCONTRER SES INVITES. ENSUITE, GAROVIRUS A DEMANDE AUX SOLDATS DE S'AMUSER. PUIS GAROVIRUS EST SORTI POUR RENCONTRER DES INVITES.

Garovirus : Amusez-vous gentiment, les enfants; Je reviens tout de suite. (ACLH: 3)

La parole (3) est le type d'acte de parole directive exigé ayant l'intention de commander. L'énoncé ou la parole montre le type d'acte de parole directive exigé car Garovirus a directement demandé à ses soldats de profiter la fête.

\section{Interdiction}

Ce type d'acte de parole directive utilise à interdire l'interlocuteur à faire quelque chose ou bien à limiter ses action.

4) CONTEXTE : LE SOIR, LORSQUE SOLDATS ET GOUVERNEURS FAISAIENT LA FETE AU PALAIS, UN OFFICIER CHARGE DE VERIFIER LES FINANCES EST VENU, MAIS L'AGENT DE SECURITE LUI A INTERDIT D'ENTRER.

La sécurité : On ne passe pas!

La parole (4) est l'acte de parole directif de type Interdiction destiné à interdire. L'énoncé s'entre au type de directif prohibitif parce que l'agent de sécurité l'interdit d'entrer au palais.

Permissif

Les permissifs sont utilisés par les locuteurs pour montrer leur confiance à l'interlocuteur lors d'une action. La confiance accordée par les interlocuteurs est une raison pour que les partenaires de parole se sentent libres d'agir. L'acte de parole aux types permissifs est comme l'approbation, la permission, l'autorisation, la concession, la concession, la permission, la permission, la libération, le pardon et la permission.

5) CONTEXTE: PENDANT LA JOURNEE, ASTERIX ET OBELIX A COMMANDE A CHERCHER DES FLEURS D'EDELWEISS EN SUISSE POUR TRAITER UNE 
INTOXICATION ALIMENTAIRE A CAIUS. ENSUITE, AU PASSAGE DE LA FRONTIERE, ILS ONT ETE ARRETES PAR DES AGENTS DE SECURITE A LA FRONTIERE. ENSUITE, ILS ONT REÇU DES QUESTIONS ET SONT EXAMINES. APRES L'AVOIR PASSE, ILS SONT AUTORISES A PASSER.

La sécurité: Ça va, Gaulois! Passez! (ACLH: 17) Guerrier de sécurité: D'accord! Parti!

La parole (5) est un acte de parole directif de types permissifs avec l'intention de permettre. Les agents de sécurité ont estimé qu'ils étaient en sécurité après avoir examiné Astérix et Obélix. Ensuite, l'agent de sécurité leur permet de continuer le voyage.

\section{Conseil}

Le caractère du type d'acte de parole directif est la confiance d'interlocuteur à ce que le locuteur dit. Dans ce cas, l'interlocuteurs time que ce que dit le locuteur est une bonne chose pour lui, et il s'agit comme le suggère le locuteur.

6) CONTEXTE: PENDANT LA JOURNEE, ASTERIX ET OBELIX DEVIENNENT LES GUIDES D'ABRARACOURCIX. ASTERIX ET OBELIX ONT DES FORMES DE CORPS DIFFERENTES. ASTERIX EST TRES PETIT ALORS QU'OBELIX EST TRES GROS. CELA FAIT RIRE LES GENS QUI LES VOIENT PARCE QU'ILS NE SONT PAS EQUILIBRES. ENSUITE, ASTERIX A SUGGERE QU'OBELIX NE NOMME QU'ABRARACOURCIX LE CHEF.

Astérix: Il vaut mieux qu'Obélix à la porte tout seul, ô chef.

La parole (6) s'entre à ce type avec l'intention de suggérer. La parole utilise des phrases déclaratives pour donner des suggestions. Les phrases déclaratives fonctionnent pour exprimer des faits, ce qui est juste, ce qui est faux, des suppositions, donner des informations, les phrases déclaratives se terminent toujours par un point (.).

\section{Forme des actes de parole directifs}

Dans cette étude, la forme des actes de parole directifs est divisée en quatre, ce sont l'acte de parole direct littérale, l'acte de parole indirect littérale, l'acte de parole direct non-littéral, l'acte de parole indirect non-littérale. Ce qui suit explique la forme des actes de langage directifs.

\section{L'acte de parole directe littéral}

L'acte de parole direct littéral est l'acte de langage qui utilise le type de phrase et le sens des mots en fonction du but recherché.

7) CONTEXTE: LE SOIR AU PALAIS, LORS QUE TOUS LES SOLDATS ET LES GOUVERNEURS JOUISSAIENT D'UNE FETE IVRE, GAROVIRUS A ORDONNE A SES SOLDATS DE FERMER LA FENETRE POUR QU'AUCUN AIR NE FENETRE DANS LA PIECE.

Fermez cette fenêtre! Ça donne l'air! (ACLH: 5)

La parole (7) est une parole directe littérale car le type de phrase et la signification des mots sont les mêmes que ceux véhiculés.. Le discours utilise des phrases impératives pour donner des ordres et se termine par un point d'exclamation. Cela indique que la commande doit être exécutée plus vite.

\section{L'acte de parole littéral indirect}

L'acte de parole indirect littéral est type de directif ayant pour but d'exprimer utilisant de phrase qui n'est pas conforme à l'intention exprimée par le locuteur, mais la signification du compilateur de phrase correspond à l'intention.

8) CONTEXTE: À CE MOMENT-LA, AU PALAIS DU GOUVERNEUR, PANORAMIX A COMMANDE SON AMI A CHERCHER DES FLEURS D'EDELWEISS, MAIS PANORAMIX A DONNE LES CONDITIONS A CAIUS, A SAVOIR QUE CAIUS SERAIT RETENU EN OTAGE JUSQU'AU RETOUR DES AMIS DE PANORAMIX.

Panoramix: Mais je ne pourrai pas le maintenir en vie très longtemps! Partez sur l'heure et faites vite! Je ferai vos adieux au village. (ACLH: 14) 
La parole (8) est un acte directif du type d'exigé (ordre), qui indique les exigences. La parole s'entre dans ce type d'acte de parole car Panoramix exige directement que Caius soit emmené dans son village en otage jusqu'au retour Astérix et Obélix. Cet énoncé est un acte de parole indirect littéral, parce que le type de phrase utilisé (déclaratif) n'est pas le même que le but exprimé (commande). Le discours littéral agit parce que le sens des mots est le même que le but exprimé.

\section{L'acte de parole direct non-littéral}

L'acte de parole directe non-littérale est l'acte de parole qui est exprimés à l'aide de types de phrases conformes aux intentions exprimées par le locuteur, mais la signification des mots qui les composent n'est pas conforme à l'objectif exprimé.

9) CONTEXTE : IL ETAIT LA MATINEE, A LA BANQUE, OU ZURIX CACHAIT ASTERIX ET OBELIX, DES SOLDATS SUISSES ONT RECHERCHE ASTERIX ET OBELIX. PUIS LE SOLDAT A VU LE COFFRE-FORT ENDOMMAGE, PUIS ZURIX A DECLARE QUE C'ETAIT ARRIVE PARCE QUE LA VOLEUR VENAIT DE SE GACHER A VOLER A LA BANQUE. ALORS LE SOLDAT ETAIT EN MARRE ET A AVERTI ZURIX DE REGARDER LE CONTENU DE SON COFFREFORT. S'IL SE PASSE QUELQUE CHOSE, IL ANNULERA TOUS LES ACCORDS CONCLUS.

Prajurit Swiss: Cette nuit, je suis occupé, mais demain matin je reviens pour annuler mon compte! Surveillez bien ce coffre là! Ce qu'il y a dedan, J'y tiens!

La parole (9) est un discours direct non littéral. L'énoncé est le type directif exigé ayant pour but de le pousser. La phrase utilisée est les phrases impératives. Dans ce cas, le soldat a un autre objectif. D'après la déclaration ci-dessus, le soldat a demandé à Zurix de faire attention au contenu de son coffre-fort. Si quelque chose se passe, il annulera son accord avec Zurix. Dans ce cas, on peut en conclure que le soldat à l' intention de pousser Zurix pour faire attention au coffre-fort.

\section{L'acte de parole indirect non-littéral}

L'acte de parole indirect non-littéral est l'acte de parole qui est exprimé dans le type de phrase et le sens qui les compose n'est pas conforme à l'intention exprimée par le locuteur.

10) CONTEXTE: A MIDI, ASTERIX A CONSEILLE OBELIX ET ABRARACOURCIX AFIN QU'OBELIX DEVIENNE LE SEUL GUIDE D'ABRARACOURCIX. ENSUITE, ABRARACOURCIX SE SENTIT UN PEU AGACE. PUIS DEMANDEZ "VOUS AVEZ REFUSE DE SERVIR LE CHEF?!"

Abraracourcix: Par toutatis! Tu refuses de me server?! (ACLH: 2)

La parole (10) est un acte indirect littéral parce que le type de phrase et le sens des mots ne sont pas les mêmes que le but exprimé. La parole utilise des phrases interrogatives mais il a le sens complet. Abraracourcix a déclaré "vous avez refusé de servir le leader?!" à Astérix et Obélix, ayant l'objectif de les maintenir sous les ordres du leader, c'est Abraracourcix. Dans ce cas, il a été conclu que la parole était l'acte de parole indirect non littéral. La phrase "Par toutatis!" est une phrase d'exclamation. Dans ce cas, Abraracourcix a exprimé sa colère contre Astérix et Obélix.

\section{CONCLUSION}

Basé sur l'analyse dans le chapitre IV, au total il y a 51 données ont été trouvés dans la bande dessinée Astérix Chez Les Helvètes. Les types et les formes d'actes de parole directif de la bande dessinée Astérix Chez Les Helvètes de René Goscinny et Albert Uderzo peuvent être classés en 6 types de discours directif et 4 formes d'actes de parole. On a trouvé quelques types d'actes de parole directif qui peuvent être classés en 1) demandé (5 données), 2) questionné (14 données), 3) exigé (25 données), 4) interdiction (2 données), 5) permissif (1 donnée), 6) conseil (4 données). À partir des résultats de l'analyse, la forme des actes de parole peut être classée en: 1) L'actes de parole directifs direct littéral(44 données), 2) L'actes de parole directifs indirect littéral(3 données), 3) L'actes de parole directifs direct non-littéral (1 donnée), 4) L'actes de parole directifs indirect non-littéral, (3 données). 
À partir de ces résultats, la chercheuse peut conclure que les actes de parole directifs qui dominent sont des types de exigé ou d'ordonnances ( 25 données et 44 données) pour des actes de parole directs littéraux. Cela montre que les personnages de la bande dessinée Astérix Chez Les Helvètes utilisent beaucoup de phrases impératives pour exprimer les commandes et les significations des mots composant la phrase conformément au but exprimé, pour faire les interlocuteurs soit facilement à comprendre la commande. Les gens qui utilise souvent la phrase impératif dans la bande dessinée Astérix Chez Les Helvètes sont les personnes qui occupent une position plus élevée, comme Abraracourcix, qui utilisent souvent des phrases impératives pour commander Astérix et Obélix

\section{BIBLIOGRAPHIE}

Altikriti, Sahar. 2016. "Persuasive Speech Acts in Barack Obama's Inaugural Speeches $(2009,2013)$ and The Last State of the Union Address (2016)". International Journal of Linguistic. Vol. 8(2). Jordan : Department of English, Faculty of Arts, Alzaytoonah University of Jordan. Bescherelle. 2006. La Grammaire Pour Tous. Paris : Hatier Bonneff, Marcel. 1998. Komik Indonesia. Terjemahan Rahayu S. Hidayat. Jakarta: KPG (Kepustakaan Populer Gramedia).

Chaer, Abdul dan Leonie Agustina. 2004. Sosiolinguistik: Perkenalan Awal. Jakarta: PT Rineka Cipta. Charlier, F.D.1975.Comment S'initier à la Linguistique?. Paris. Librairie Larousse.

Ciptaningtyas, Ika Septian. 2012. "Tindak Tutur Direktif dalam Film Astérix et Obélix Mission Cléopâtra". Skripsi. Yogyakarta : Universitas Negeri Yogyakarta.

Goddard, Cliff. 2002. "Directive speech acts in Malay (Bahasa Melayu) : an ethnopragmatic perspective Actes de parole directifs en malais : une perspective ethnopragmatique." Cahiers de Praxématique. Australia. University of New England.

Handayani, Conny, Wahyudi J.S, dan Diah V.W. 2011. "Tindak Tutur Direktif Dosen Dengan Tenaga Administrasi: Ancangan Sosiopragmatik Berspektif Jender." Lingua. Vol.7(2). Semarang : Universitas Negeri Semarang. Ibrahim, Abd. Syukur. 1993. Kajian Tindak Tutur. Surabaya: Usaha Nasional.

Kridalaksana, Harimurti. 2008. Kamus Linguistik. Jakarta : Gramedia Pustaka Utama.

Kurniasih. 2017. "Tindak Tutur Direktif Dalam Dialog Film Animasi Mofy. Skripsi." Semarang : Universitas Negeri Semarang.

Leech, Geofrey. 1993. Principles of Pragmatics. London dan New York: Longman. Lubis, Hasan. 2010. Analisis Wacana Pragmatik. Bandung : Angkasa.

Maharani. 2007. "Tindak Tutur dalam Percakapan dalam Komik Astérix. Skripsi. Medan." Universitas Sumatra Utara.

Malau, Farida. 2009. "Tindak Tutur dalam Seri Cerita Kenangan Agenteul Hidup Memisahkan Diri Karya N.H.Dini." Skripsi. Medan. Universitas Sumatra Utara.

Morris, Charles. 1938. Foundation of The Theory of Signs. Chicago: University of Chicago Press.

Muaya, Kristle Priskila. 2014. "Bentuk Dan Fungsi Tindak Tutur Direktif Pada Komik Lucky Luke Volume 22 Les Dalton Dans Le Blizzard Karya Morris Dan R. Goscinny." Skripsi. Yogyakarta : Universitas Negeri Yogyakarta.

Rohmadi, Muhammad. 2004. Analisis Wacana Pragmatik (Kajian Teori dan Analisisnya). Surakarta : Yuma Pustaka.

Rustono. 1999. Pokok-pokok Pragmatik. Semarang : CV. IKIP Semarang.

Santoso, Agil Hari. 2018. "Tindak Tutur Ilokusi Pada Film Intouchables Karya Éric Toledano Dan Olivier Nakache." Skripsi. Semarang : Universitas Negeri Semarang.

Sudaryanto. 2015. Metode dan Aneka Teknik Analisis Bahasa.Yogyakarta : Duta Wacana University.

Wijana, I Dewa Putu. 1996. Dasar-dasar Pragmatik. Yogyakarta: Andi Yogyakarta.

Wijana, I Dewa Putu. 2004. Pragmatik:Teori dan Analisis. Yogyakarta:Lingkar Media.

Yule, George. 2006. Pragmatik. Terjemahan oleh Indah Fajar Wahynni. Yogyakarta: Pustaka Pelajar. 